\title{
The Acoustic Design of Soundproofing Doors and Windows
}

\author{
${ }^{I}$ Kumamoto National College of Technology, 2659-2 Suya Koshi shi Kumamoto, Japan \\ ${ }^{2}$ Faculty of Engineering, Kumamoto University, 2-39-1 Kurokami Kumamoto, Japan \\ ${ }^{3}$ Faculty of Computer Science, Sojo University, 4-22-1 Ikeda Kumamoto, Japan
}

Nishimura Yuya ${ }^{*}, 1$, Nguyen Huy Quang ${ }^{2}$, Nishimura Sohei ${ }^{1}$, Nishimura Tsuyoshi ${ }^{3}$ and Yano Takashi ${ }^{2}$

\begin{abstract}
A model for manufacturing doors and windows which are capable of natural ventilating, reducing traffic noise and so on for the developing tropical countries is presented. These windows and doors combine two basic components which are ventilation unit and lighting unit. Due to the fact that the ventilation unit must have a large volume to attenuate low frequency noise, many resonance of higher-order mode wave will be generated inside the unit. In this work, a method to predict the insertion-loss of rectangular ventilation unit with input and output openings at various positions is proposed by solving the wave equation, considering the resonance frequencies of higher-order mode. The results of the analysis have been confirmed by experiments.
\end{abstract}

Keywords: Soundproofing, resonance frequency, casement windows, higher-order modes.

\section{INTRODUCTION}

Sealing up type's doors and windows are widely used in the current houses to intercept an inside outside. Needless to say, some equipments such as air conditioners, are necessary to keep a comfortable indoor temperature while such doors and windows are closed and the using duration of such equipments is often limited because of the power consumption cost and health. The authors have been presented a concept for manufacturing windows which are capable of ventilating, regulating sunlight and reducing traffic noise for the developing tropical countries [1]. These windows combine two basic components which are ventilation unit and lighting unit. The former also serves as an import function in reducing noise.

Due to the fact that the ventilation unit must have a large volume to attenuate low-frequency noise, many resonance of higher-order mode wave will be generated inside the unit [25]. Consequently, it is necessary to take into consideration the selection of size and placement of input and output openings in such a way that would minimize the effects of higher-order mode in order to have a great soundproofing effect. In the previous analysis, the ventilation unit is constructed using rectangular cavity with input and output openings at both ends. Actually, an input and output can be located at various positions on the unit according to the door or window's design. Moreover, the optimum location where an insertion loss effect is effectively achieved is still an unknown problem.

In this article, a method to predict the insertion loss of rectangular cavity with input and output openings at various positions is proposed. Following the development of the

\footnotetext{
*Address correspondence to this author at the Kumamoto National College of Technology, 2659-2 Suya Koshi shi Kumamoto, Japan; Tel: +81-963263605; Fax: +81-96-3263605; E-mail: nisimura@cis.sojo-u.ac.jp
}

theory in section 3 , this is compared to experimental results in section 4 , an excellent agreement is obtained.

\section{DESIGN OF SOUNDPROOFING WINDOWS/ DOORS AND THE VENTILATION UNIT}

Casement windows and doors are consisted of one or several wooden frames that can be opened and closed at various angles and are widely used in countries with tropical climates. An example of casement door's structure is shown in Fig. (1). The windows and doors are opened during day for naturally ventilating the room and closed at night, even when closed room ventilation is still achieved because the windows and doors are constructed with a lots of ventilating slits. However, an annual increase in traffic noise level these countries have rendered these windows and doors to be useless because these ventilating slits serve as a direct pathway for traffic noise to enter the home.

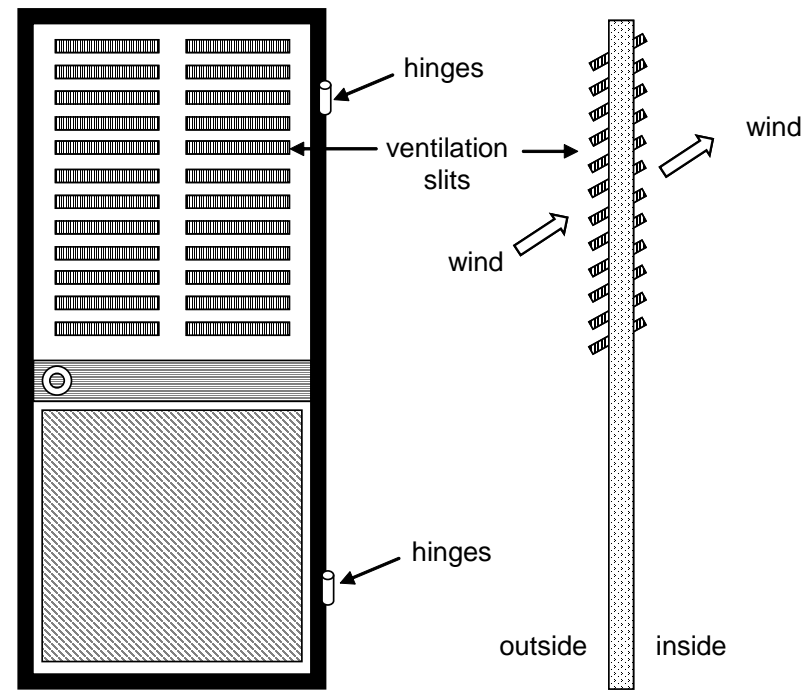

Fig. (1). The casement doors of current houses in tropical climate countries. 
Based on the previous reports, our proposed casement's door is shown in Fig. (2). It combines two basic components: ventilation and lighting. The lighting unit can be constructed using one or two glass layers which are mounted between two ventilation components with input and output openings as shows in Fig. (3). The ventilation unit may consist of square, rectangular or more complicated shapes depending on decorative considerations. Needless to say, the unit requires a simple internal structure and large input and output to maximize ventilation, as well as preventing outside noise from entering the home. Actually, an input and output can be located at various positions on the cavity according to the door or window design. Therefore, these combinations are considered as follows:

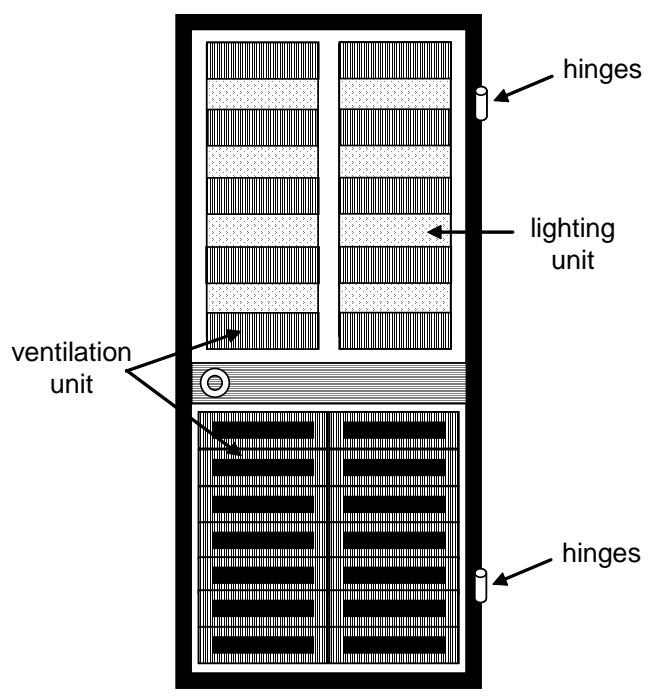

Fig. (2). The proposed casement doors.

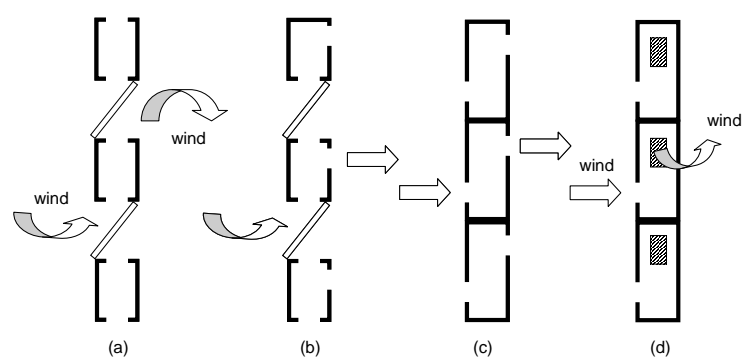

Fig. (3). Design of the proposed casement windows.

Combinations of input and output positions are shown in Fig. (4) in which (a) and (b) are the cases where they are in opposite faces while, (c) and (d) are in faces which crossed right angles. Difference between (a) and (b) is whether they are located in big or small cavity area, however it is quite similar in the theoretical analysis. Hereafter, we name (a) and (b) as Model-1.

Similarly, difference between (c) and (d) is whether they are located in big or small side area which crossed right angles. In this analysis, once we find the acoustic characteristic when the input and output are located in arbitrary face, it is easily computable for other faces by exchanging coordinates. We name (c) and (d) as Model-2. The sound propagation in (a) is the same as (c) and that in (b) is the same as (d).

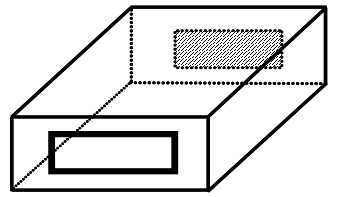

(a)

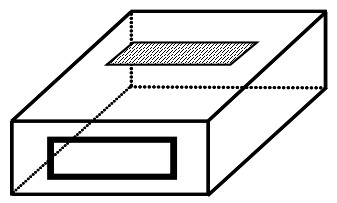

(c)

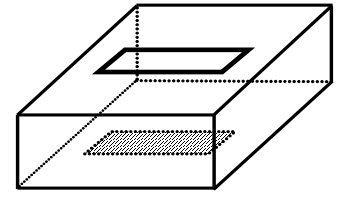

(b)

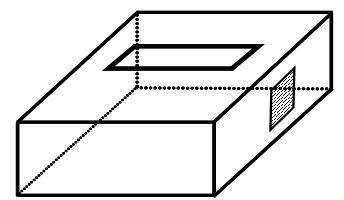

(d)
Fig. (4). Combinations of input and output positions on rectangular cavity.

\section{METHOD OF ANALYSIS}

\subsection{Insertion Loss}

Acoustic characteristics of an acoustic element can be described by the four-pole parameters A, B, C and D as [6]

$\left[\begin{array}{l}P_{1} \\ U_{1}\end{array}\right]=$

$\left[\begin{array}{cc}A & B \\ C & D\end{array}\right]\left[\begin{array}{c}P_{2} \\ U_{2}\end{array}\right]=\left[\begin{array}{cc}\cos k l & j Z \sin k l \\ j \frac{1}{Z} \sin k l & \cos k l\end{array}\right]\left[\begin{array}{c}P_{2} \\ U_{2}\end{array}\right]$

where $P_{1}$ and $U_{1}$ are the sound pressure and velocity at the input, $P_{2}$ and $U_{2}$ are at the output, $Z$ is the characteristic impedance, $k$ is the wave number and $l$ is the length. When this element is connected to the source, their performance can be expressed through the use of insertion loss $I L$ defined by [6]

$I L=10 \log \frac{W_{r}}{W_{0}}=10 \log \left|\frac{U_{1}}{U_{2}}\right|^{2}$

Here, $W_{r}$ and $W_{0}$ are the radiated power at one point in space with or without the acoustic element inserted between that point and the source. The ratio of $U_{1} / U_{2}$ is equal to the $D$ parameter of Eq. (1), as far as constant velocity source is concerned.

When the acoustic element is connected in series as shown in Fig. (5), the sectional area of element 1 and 3 becomes significantly small as compared to the sectional area of element 2 and the $D$ parameter of whole system can be described by the following approximate equation

$D=\left(\cos k l_{1}\right)\left(C_{w}\right)\left(j Z_{3} \sin k l_{3}\right)$ 
where $C_{w}$ denoted the $\mathrm{C}$ parameter of element 2. As shown in Eq. (2) and (3), in order to obtain a reliable $I L$ effect, $D$ parameter must be high enough. In other words, the design of element-2 to have a high enough parameter $C_{w}$ is demanded.

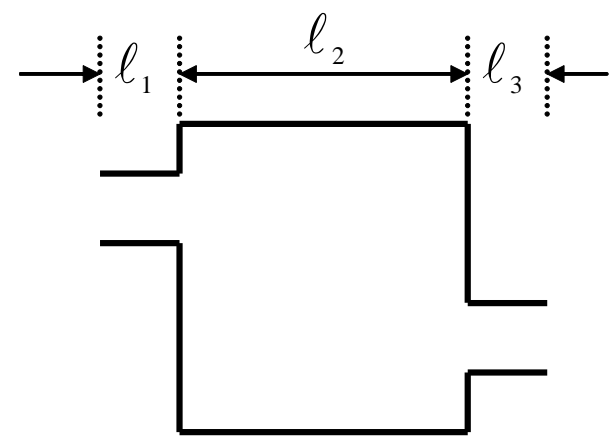

Fig. (5). Basic structure of ventilation unit.

\subsection{Computation of $C_{w}$}

First of all, we find the sound pressure on the input and output of the Model-1 as shown in Fig. (6a). The input and output have a sectional area of $S_{i}=2 \alpha_{i} \times 2 \beta_{i}$ and $S_{0}=2 \alpha_{0} \times 2 \beta_{0}$ where its center is located at point $I\left(x_{i}, y_{i}, 0\right)$ and $O\left(x_{0}, y_{0}, L\right)$, respectively.

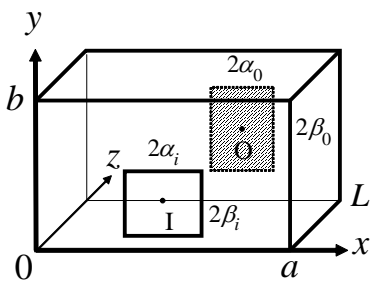

(a)

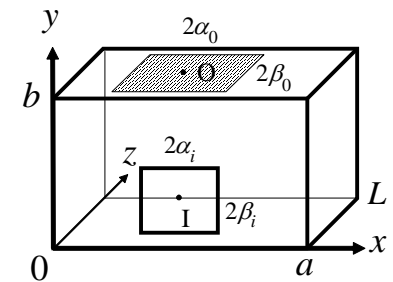

(b)
Fig. (6). Model of computation.

The complete wave-equation in terms of the velocity potential $\phi$ when expressed in rectangular coordinates is given by [1]

$\phi=\left(A e^{\mu z}+B e^{-\mu z}\right)(C \sin \alpha x+D \cos \alpha x)$

$\left(E \sin \sqrt{s^{2}-\alpha^{2}} y+F \cos \sqrt{s^{2}-\alpha^{2}} y\right)$

where $A, B, C, D, E$ and $\mathrm{F}$ are arbitrary constants determinable from the boundary conditions, $\alpha, s$ and $\mu$ are the constants.

Let $\quad V_{x}=-\partial \phi / \partial x, \quad V_{y}=-\partial \phi / \partial y$ and $V_{z}=-\partial \phi / \partial z$ be the velocity components in the $\mathrm{x}, \mathrm{y}$ and $\mathrm{z}$ directions, respectively. Assuming the walls of the cavity to be perfectly rigid and the loss at the wall can be neglected, the boundary conditions are

[1] at $x=0, V_{x}=0$

[2] at $x=a, V_{x}=0$
[3] at $y=0, V_{y}=0$

[4] at $y=b, V_{y}=0$

[5] at $z=0, V_{z}=V_{i} F_{i}(x, y)$

[6] at $z=L, V_{z}=0$

where $V_{i}$ is the driving velocity at the input, $F_{i}(x, y)$ is the function which has a value of 1 at the input section area and 0 at the other area, namely $\left(x_{i}-\alpha_{i} \leq x \leq x_{i}+\alpha_{i}\right.$, $\left.y_{i}-\beta_{i} \leq y \leq y_{i}+\beta_{i}\right)$

At first, Eq. (5) gives

$\left.\frac{\partial}{\partial x}(C \sin \alpha x+D \cos \alpha x) \quad\right|_{x=0}=0 \quad \therefore \quad C=0$

And from Eq. (6)

$\left.\frac{\partial}{\partial x} D \cos \alpha x \quad\right|_{x=a}=0 \therefore \alpha=m \frac{\pi}{a}(m=0,1,2, \ldots)$

Substituting Eqs. (11) and (12) into Eq. (4) gives

$\phi=\sum_{m=0}^{\infty}\left(A e^{\mu z}+B e^{-\mu z}\right) \cos \left(m \frac{\pi}{a}\right)$

$\left(E \sin \sqrt{s^{2}-\alpha^{2}} y+F \cos \sqrt{s^{2}-\alpha^{2}} y\right)$

from Eq. (7)

$\left.\frac{\partial}{\partial y}\left(E \sin \sqrt{s^{2}-\alpha^{2}} y+F \cos \sqrt{s^{2}-\alpha^{2}} y\right)\right|_{y=0}=0 \therefore E=0$

and from Eq. (8)

$\left.\frac{\partial}{\partial y}\left(F \cos \sqrt{s^{2}-\alpha^{2}} y\right)\right|_{y=b}=0 \therefore \sqrt{s^{2}-\alpha^{2}}=n \frac{\pi}{b}(n=0,1,2, \ldots)$

Substituting Eqs. (14) and (15) into Eq. (13), so $\phi$ becomes

$\phi=\sum_{m=0}^{\infty} \sum_{n=0}^{\infty}\left(A e^{\mu_{m, n} z}+B e^{-\mu_{m, n} z}\right) \cos \left(\frac{m \pi x}{a}\right) \cos \left(\frac{n \pi y}{b}\right)$

where

$\mu_{m, n}=\sqrt{(m \pi / a)^{2}+(n \pi / b)^{2}-k^{2}}$

Next, from Eq. (10)

$\left.\frac{\partial}{\partial z}\left(A e^{\mu_{m, n} z}+B e^{-\mu_{m, n} z}\right) \quad\right|_{z=L}=0 \quad \therefore B=A e^{2 \mu_{m, n} L}$

Substituting $B$ into Eq. (16) yields

$\phi=\sum_{m=0}^{\infty} \sum_{n=0}^{\infty} e^{\mu_{m, L} L} \cosh \left(\mu_{m, n}(z-L)\right) C_{m, n} \cos \left(\frac{m \pi x}{a}\right) \cos \left(\frac{n \pi y}{b}\right)$

from Eq. (9) 
$-\frac{\partial}{\partial z} \sum_{m=0}^{\infty} \sum_{n=0}^{\infty} e^{\mu_{m, n} L} \cosh \left(\mu_{m, n}(z-L)\right) C_{m, n} \cos \left(\frac{m \pi x}{a}\right)$

$\left.\cos \left(\frac{n \pi y}{b}\right) \quad\right|_{z=0}=V_{i} F_{i}(x, y)$

By multiplying both sides of Eq. (20) by $\cos (m \pi x / a) \cos (n \pi y / b)$ and integrating with respect to $x$ from 0 to $a$ and with respect to $y$ from 0 to $b, C_{m, n}$ can be determined.

Substitution of $C_{m, n}$ to (19) yields

$\phi=\sum_{m=0}^{\infty} \sum_{n=0}^{\infty} \frac{\cosh \left(\mu_{m, n}(z-L)\right)}{\mu_{m, n} \sinh \left(\mu_{m, n} L\right)} U_{i} D_{m, n} \cos \left(\frac{m \pi x}{a}\right) \cos \left(\frac{n \pi y}{b}\right)$

where

$D_{m, n}=\frac{16}{m n \pi^{2} S_{i}} \cos \left(\frac{m \pi x_{i}}{a}\right) \cos \left(\frac{n \pi y_{i}}{b}\right) \sin \left(\frac{m \pi \alpha_{i}}{a}\right) \sin \left(\frac{n \pi \beta_{i}}{b}\right)$

$U_{i}=V_{i} S_{i}$ is the volume velocity at input.

Therefore, sound pressure corresponding to $\phi$ becomes

$P(x, y, z)=j k \rho c \phi$

$=j k Z_{w} U_{i} S_{w} \sum_{m=0}^{\infty} \sum_{n=0}^{\infty} \frac{\cosh \left(\mu_{m, n}(z-L)\right)}{\mu_{m, n} \sinh \left(\mu_{m, n} L\right)} D_{m, n} \cos \left(\frac{m \pi x}{a}\right) \cos \left(\frac{n \pi y}{b}\right)$

where $Z_{w}=\rho c / S_{w}$ represents the characteristic acoustic impedance of the cavity.

The sound pressure at the input side $z=0$ becomes

$P_{i}=P(x, y, 0)=$

$j k Z_{w} U_{i} S_{w} \sum_{m=0}^{\infty} \sum_{n=0}^{\infty} \frac{\cosh \left(\mu_{m, n} L\right)}{\mu_{m, n} \sinh \left(\mu_{m, n} L\right)} D_{m, n} \cos \left(\frac{m \pi x}{a}\right) \cos \left(\frac{n \pi y}{b}\right)$

Therefore, the average sound pressure on the input is given by

$\bar{P}_{i}=\frac{1}{S_{i}} \int_{x_{i}-\alpha_{i}}^{x_{i}+\alpha_{i}} \int_{y_{i}-\beta_{i}}^{y_{i}+\beta_{i}} P(x, y, 0) d x d y$

$=j k Z_{w} U_{i} \sum_{m=0}^{\infty} \sum_{n=0}^{\infty} \frac{\cosh \left(\mu_{m, n} L\right)}{\mu_{m, n} \sinh \left(\mu_{m, n} L\right)} \bar{E}_{m, n}$

where the level $\bar{E}_{m, n}$ corresponding to $(m, n)$ modes is given as

$$
\bar{E}_{m, n}=\frac{S_{w}}{S_{i}} D_{m, n} \int_{x_{i}-\alpha_{i}}^{x_{i}+\alpha_{i}} \int_{y_{i}-\beta_{i}}^{y_{i}+\beta_{i}} \cos \left(\frac{m \pi x}{a}\right) \cos \left(\frac{n \pi y}{b}\right) d x d y
$$

Similarly, by setting $z=L$ in Eq. (23), the sound pressure on the output becomes

$P_{0}=P(x, y, L)=$

$j k Z_{w} U_{i} S_{w} \sum_{m=0}^{\infty} \sum_{n=0}^{\infty} \frac{1}{\mu_{m, n} \sinh \left(\mu_{m, n} L\right)} D_{m, n} \cos \left(\frac{m \pi x}{a}\right) \cos \left(\frac{n \pi y}{b}\right)$ and the average sound pressure on the output becomes

$$
\begin{aligned}
\bar{P}_{0} & =\frac{1}{S_{0}} \int_{x_{0}-\alpha_{0}}^{x_{0}+\alpha_{0}} \int_{y_{0}-\beta_{0}}^{y_{0}+\beta_{0}} P(x, y, L) d x d y \\
& =j k Z_{w} U_{0} \sum_{m=0}^{\infty} \sum_{n=0}^{\infty} \frac{\bar{Q}_{m n}}{\mu_{m, n} \sinh \left(\mu_{m, n} L\right)}
\end{aligned}
$$

where

$\bar{Q}_{m, n}=\frac{S_{w}}{S_{0}} D_{m, n} \int_{x_{0}-\alpha_{0}}^{x_{0}+\alpha_{0}} \int_{y_{0}-\beta_{0}}^{y_{0}+\beta_{0}} \cos \left(\frac{m \pi x}{a}\right) \cos \left(\frac{n \pi y}{b}\right) d x d y$

Moreover, expanding Eq. (28) corresponding to the variation of $m$ and $n$, we have

$$
\begin{aligned}
& \bar{P}_{0}=j Z_{w} U_{i}\left\{-\frac{1}{\sin (k L)}+\sum_{m=1}^{\infty} \frac{\bar{Q}_{m, 0}}{\mu_{m, 0} \sinh \left(\mu_{m, 0} L\right)}+\sum_{n=1}^{\infty} \frac{\bar{Q}_{0, n}}{\mu_{0, n} \sinh \left(\mu_{0, n} L\right)}\right. \\
& \left.+\dot{\sum} \frac{\bar{Q}_{m, n}}{\mu_{m, n} \sinh \left(\mu_{m, n} L\right)}\right\}
\end{aligned}
$$

where

$$
\begin{aligned}
& \bar{Q}_{m, 0}=\frac{S_{w}}{S_{0}} \frac{4 \beta_{0} a}{m \pi} D_{m, 0} \cos \left(\frac{m \pi x_{0}}{a}\right) \sin \left(\frac{m \pi \alpha_{0}}{a}\right) \\
& \bar{Q}_{0, n}=\frac{S_{w}}{S_{0}} \frac{4 \alpha_{0} b}{n \pi} D_{0, n} \cos \left(\frac{n \pi y_{0}}{b}\right) \sin \left(\frac{n \pi \beta_{0}}{b}\right) \\
& D_{m, 0}=\frac{8 \beta_{i}}{m \pi S_{i} b} \cos \left(\frac{m \pi x_{i}}{a}\right) \sin \left(\frac{m \pi \alpha_{i}}{a}\right) \\
& D_{0, n}=\frac{8 \alpha_{i}}{n \pi S_{i} a} \cos \left(\frac{n \pi y_{i}}{b}\right) \sin \left(\frac{n \pi \beta_{i}}{b}\right)
\end{aligned}
$$

the symbol $\dot{\sum}$ means $\sum_{m=0}^{\infty} \sum_{n=0}^{\infty}$ without the cases of $m$ and $n$ in 1 st -3 rd term of Eq. (30).

The $C_{w}$ can be obtain by $C_{w}=U_{i} / \bar{P}_{0}$ under the condition of the output velocity of the volume, where $U_{0}=V_{z} S_{0}$ is equal to zero. Note that, this condition has already been considered in Eq. (10).

In the case of Model-2 as shown in Fig. (6b), the input and output have a sectional area of $S_{i}=2 \alpha_{i} \times 2 \beta_{i}$ and $S_{0}=2 \alpha_{0} \times 2 \beta_{0}$ where its center is located at point $I\left(x_{i}, y_{i}, 0\right)$ and $O\left(x_{0}, b, z_{0}\right)$, respectively.

After the similar computation procedure is performed from Eq. (4) to Eq. (23), the sound pressure on the side where the output located can be derived as

$$
\begin{aligned}
& P_{0}=P(x, b, z)=j k Z_{w} U_{i} S_{w} \sum_{m=0}^{\infty} \sum_{n=0}^{\infty} \frac{\cosh \left(\mu_{m, n}(z-L)\right)}{\mu_{m, n} \sinh \left(\mu_{m, n} L\right)} D_{m, n} \\
& \cos \left(\frac{m \pi x}{a}\right) \cos (n \pi)
\end{aligned}
$$


Therefore, the average output sound pressure becomes

$\bar{P}_{0}=\frac{1}{S_{0}} \int_{x_{0}-\alpha_{0}}^{x_{0}+\alpha_{0}} \int_{z_{0}-\beta_{0}}^{z_{0}+\beta_{0}} P(x, b, z) d z d y$

$=j k Z_{w} U_{i} \frac{S_{w}}{S_{0}} \sum_{m=0}^{\infty} \sum_{n=0}^{\infty} \frac{D_{m, n} \cos (n \pi)}{\mu_{m, n} \sinh \left(\mu_{m, n} L\right)}$

$\int_{x_{0}-\alpha_{0}}^{x_{0}+\alpha_{0}} \int_{z_{0}-\beta_{0}}^{z_{0}+\beta_{0}} \cosh \left(\mu_{m, n}(z-L)\right) \cos \left(\frac{m \pi x}{a}\right) d z d x$

$=j k Z_{w} U_{i} \sum_{m=0}^{\infty} \sum_{n=0}^{\infty} \frac{\bar{R}_{m, n}}{\mu_{m, n} \sinh \left(\mu_{m, n} L\right)}$

where

$\bar{R}_{m, n}=$

$\frac{S_{w}}{S_{0}} D_{m, n} \cos (n \pi) \int_{x_{0}-\alpha_{0}}^{x_{0}+\alpha_{0}} \int_{z_{0}-\beta_{0}}^{z_{0}+\beta_{0}} \cosh \left(\mu_{m, n}(z-L)\right) \cos \left(\frac{m \pi x}{a}\right) d z d x$

Moreover, expanding the above equation corresponding to variation of $m$ and $n$, we have

$\bar{P}_{0}=j Z_{w} U_{i}\left\{-\frac{\bar{R}_{0,0}}{k \sin (k L)}+\sum_{m=1}^{\infty} \frac{\bar{R}_{m, 0}}{\mu_{m, 0} \sinh \left(\mu_{m, 0} L\right)}\right.$

$\left.+\sum_{n=1}^{\infty} \frac{\bar{R}_{0, n}}{\mu_{0, n} \sinh \left(\mu_{0, n} L\right)}+\dot{\sum} \frac{\bar{R}_{m, n}}{\mu_{m, n} \sinh \left(\mu_{m, n} L\right)}\right\}$

where

$\bar{R}_{0,0}=-\frac{1}{S_{0}} \int_{x_{0}-\alpha_{0}}^{x_{0}+\alpha_{0}} \int_{z_{0}-\beta_{0}}^{z_{0}+\beta_{0}} \cos (k(z-L)) d z d x$

$=-\frac{1}{S_{0}} \frac{2 \alpha_{0}}{k}\left\{\sin \left(k\left(\beta_{0}+L-z_{0}\right)+\sin \left(k\left(\beta_{0}-L+z_{0}\right)\right\}\right.\right.$

Finally, $C_{w}$ can be derive as

$C_{w}=\frac{U_{i}}{\bar{P}_{0}}$

\section{RESULT AND DISCUSSION}

$C_{w}$ is defined by Eq. (40) including the average output sound pressure $\bar{P}_{0}$ at its denominator, where $\bar{P}_{0}$ is given in Eq. (30) and Eq. (38) for Model-1 and Model-2, respectively. Note that both models have the same average input sound pressure $\bar{P}_{i}$ defined by Eq. (25). In order to obtain an IL effectively $C_{w}$ must be at great, in other words, low level of $\bar{P}_{0}$ is preferable. Referring to Eq. (30), $\bar{P}_{0}$ becomes great when its denominator $\sin (k L)$ and $\mu_{m, n} \sinh \left(\mu_{m, n} L\right)$ are zero, namely, at the following resonance frequencies of

$$
\begin{aligned}
& \sin (k L)=0 \quad \therefore \quad f_{0}=\eta \frac{c}{2 L} \quad(\eta=1,2,3 \ldots) \\
& \mu_{m, n} \sinh \left(\mu_{m, n} L\right)=0 \quad \therefore \quad f_{m, n}=\frac{c}{2 \pi} \sqrt{\left(\frac{m \pi}{a}\right)^{2}+\left(\frac{n \pi}{b}\right)^{2}+\left(\frac{\eta \pi}{L}\right)^{2}} \\
& (\eta=0,1,2,3 \ldots)
\end{aligned}
$$

The $f_{0}$ represents the resonance frequencies of the plane wave and $f_{m, n}$ represents those of traverse wave. The first resonance frequencies of $f_{m, n}$ when $\eta=0$ occur sequentially, are shown in Table $\mathbf{1}$, when the dimensions of the cavity are $a=0.48 \mathrm{~m}, b=0.075 \mathrm{~m}$ and $L=0.29 \mathrm{~m}$, respectively. Generally, at the frequency range where the traverse waves are generated, the sound pressure $\bar{P}_{0}$ will increase and when the resonance frequencies of other modes co-occur, $C_{w}$ will be small and the $I L$ can not be expected to be as great. Generation mechanism of these frequencies can be understood according to the calculation example as shown in Fig. (7a) with $(2,0)$ and $(4,0)$ modes. Dimensions of the cavity used in calculation are the same as in Table $\mathbf{1 .}$

Table 1. The First Resonance Frequency of Some $(m, n)$ Mode Occur in the Cavity when $a=0.48 \mathrm{~m}, b=0.075 \mathrm{~m}$ and $L=0.29 \mathrm{~m}$

\begin{tabular}{|c|c|c|}
\hline \multicolumn{2}{|c|}{ Mode } & 1st Resonance \\
\hline $\mathbf{m}$ & $\mathbf{n}$ & Frequency (Hz) \\
\hline \hline 1 & 0 & 369 \\
\hline 2 & 0 & 739 \\
\hline 3 & 0 & 1109 \\
\hline 4 & 0 & 1479 \\
\hline 5 & 0 & 1848 \\
\hline 6 & 0 & 2218 \\
\hline 0 & 1 & 2366 \\
\hline 1 & 1 & 2395 \\
\hline 2 & 1 & 2479 \\
\hline 7 & 0 & 2588 \\
\hline 3 & 1 & 2613 \\
\hline 4 & 1 & 2790 \\
\hline 8 & 0 & 2958 \\
\hline
\end{tabular}

Sound pressure level of those wave components in $\mathrm{dB}$ are shown in Fig. (7b). They also have many resonance frequencies which occur corresponding to the increasing of $\eta$ in Eq. (42). Therefore, it is clear that when we eliminate an arbitrary higher-order wave mode by any method, we will not only avoid many resonances generated by this mode but also obtain the low level of the entire output sound pressure. 


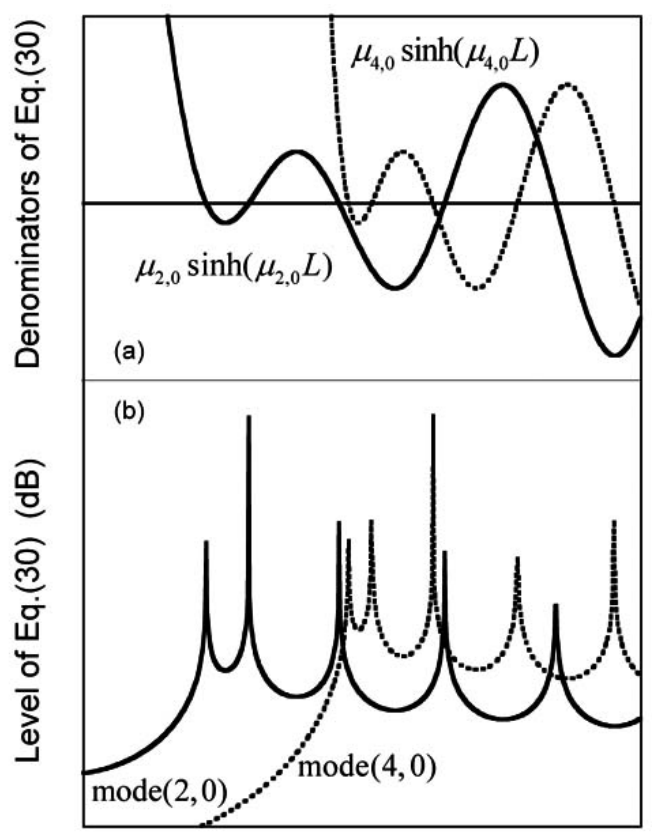

Frequency $(\mathrm{Hz})$

Fig. (7). Physical meaning of Eq. (30). (a) Calculated example of Eq. (30) with $(2,0)$ mode and $(4,0)$ mode. Resonances will occur when these denominators become zero. Dimensions of cavity are $a=0.48 \mathrm{~m}, b=0.075 \mathrm{~m}$ and $L=0.29 \mathrm{~m}$. (b) Spectrum of $(2,0)$ mode, $(4,0)$ mode.

Next, in order to examine the accuracy of our predicted result, the measurement in some positions on the cavity was carried out by using the previous measurement method (see Appendix).

Measurement result of $C_{w}$ of Model-1 when the input and output are located in the center position $(a / 2, b / 2)$ is shown in Fig. (8b), while the denominators of Eq. (30) are shown in Fig. (8a). Agreement observed between the measurement and our predicted resonance frequencies is acceptable. Note that, by locating the input or output at $x=a / 2$ and $y=b / 2, \quad \cos (m \pi x / a) \cos (n \pi y / b)$ in Eq. (27) will become $\cos (m \pi / 2) \cos (n \pi / 2)$ and as a result, the $(m, n)$ mode sound pressure does not appear when $m=1,3,5 \ldots$ for all values of $\mathrm{n}$ and when $n=1,3,5 \ldots$ for all values of $m$. Therefore, as shown in Fig. $(\mathbf{8 b})$, the $(m, n)$ mode sound pressures appears in cavity in the order of $(2,0),(4,0)$ and so on.

Fig. (9) shows the measured and theoretical results of Model-2 at point A $(a / 2, b, L / 2)$ located on the top of the cavity. By locating at $z=L / 2$, Eq. (35) becomes

$$
\begin{aligned}
& P_{A}=P(a / 2, b, L / 2)= \\
& j k Z_{w} U_{i} S_{w} \sum_{m=0}^{\infty} \sum_{n=0}^{\infty} \frac{\cosh \left(\mu_{m, n} L / 2\right)}{\mu_{m, n} \sinh \left(\mu_{m, n} L\right)} D_{m, n} \cos \left(\frac{m \pi}{2}\right) \cos (n \pi) \\
& =j k Z_{w} U_{i} S_{w} \frac{1}{2} \sum_{m=0}^{\infty} \sum_{n=0}^{\infty} \frac{1}{\mu_{m, n} \sinh \left(\mu_{m, n} L / 2\right)} D_{m, n} \\
& \cos \left(\frac{m \pi}{2}\right) \cos (n \pi)
\end{aligned}
$$

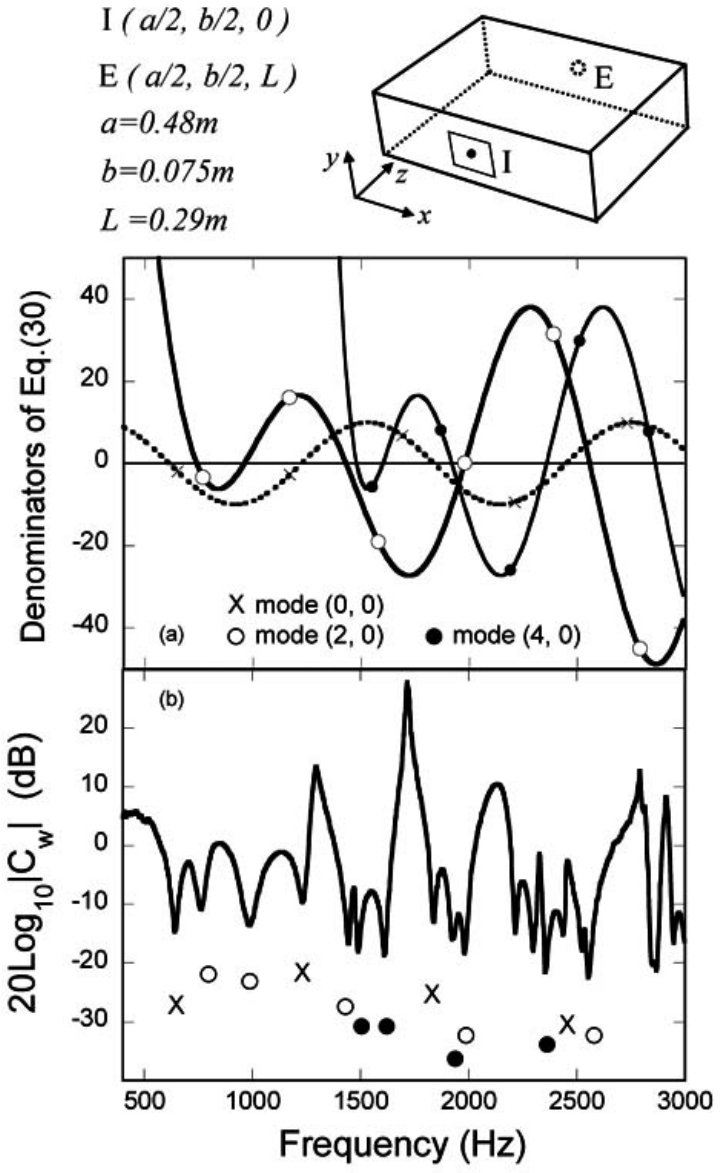

Fig. (8). Resonance frequencies appear in the cavity when the output is located at position E. (a) computed result of the denominator of Eq. (30). (b) measured result of $C_{w}$.

Moreover, expanding the above equation with mode $(0,0)$ we have

$P_{A}=j k Z_{w} U_{i} S_{w} \frac{1}{2}\left\{\begin{array}{l}\frac{1}{k \sin (k L / 2)}+\dot{\sum} \frac{1}{\mu_{m, n} \sinh \left(\mu_{m, n} L / 2\right)} D_{m, n} \\ \cos \left(\frac{m \pi}{2}\right) \cos (n \pi)\end{array}\right\}$

As a result, the denominators of $P_{A}$ become $\sin (k L / 2)$ and $\sinh \left(\mu_{m, n} L / 2\right)$, therefore, the resonance frequencies will differ from those of Fig. (8). Calculation examples of $\operatorname{mode}(0,0)$ and $\operatorname{mode}(4,0)$ are shown in Fig. (9b). The frequencies at which $\sin (k L / 2)$ and $\sinh \left(\mu_{m, n} L / 2\right)$ become zero correspond well to those of the measured results shown in Fig. (9a). In order to show the difference among the resonance frequencies, the measured result at position $\mathrm{E}$, which had been described above, was added to Fig. (9a).

Fig. (10) shows the measured result at point $\mathrm{C}(a, b / 2$, $L / 2$ ) that locates in the right side of the cavity. Because the resonance frequencies are the same as point $\mathrm{A}$ as described above, the effectiveness of our calculation method has been proven. 


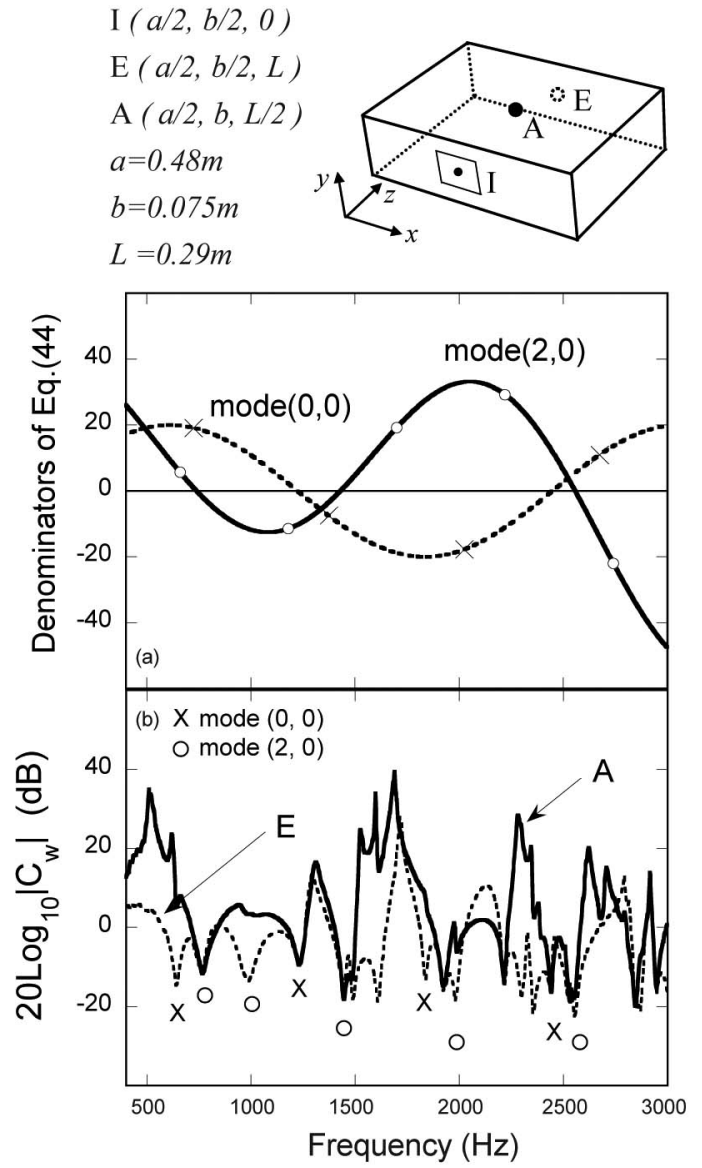

Fig. (9). Resonance frequencies appear in the cavity when the output is located at position A and E. (a) computed result of the denominator of Eq. (44) (b) measured result of $C_{w}$.

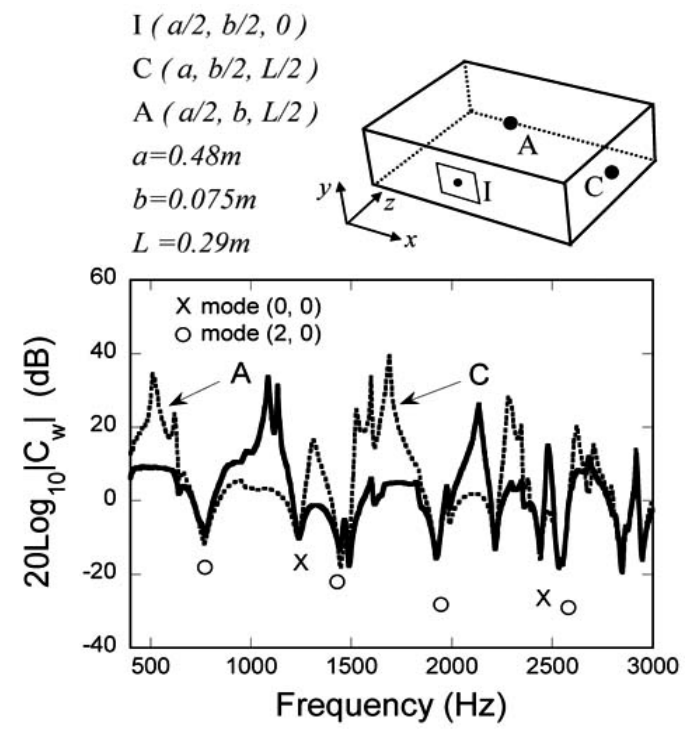

Fig. (10). Measured results at position $A$ and $C$.

Fig. (11) shows the theoretical and measured results of Model-1 at point A $(a / 2, b, L / 2)$ which locates on the top of the cavity when the input located on the opposite face. In this case, the first resonance frequencies of $f_{m, n}$ that occur sequentially are shown in Table 2 . Because the input was located on the big cavity area, resonance frequencies of higher-order mode generally appear in low frequency range.

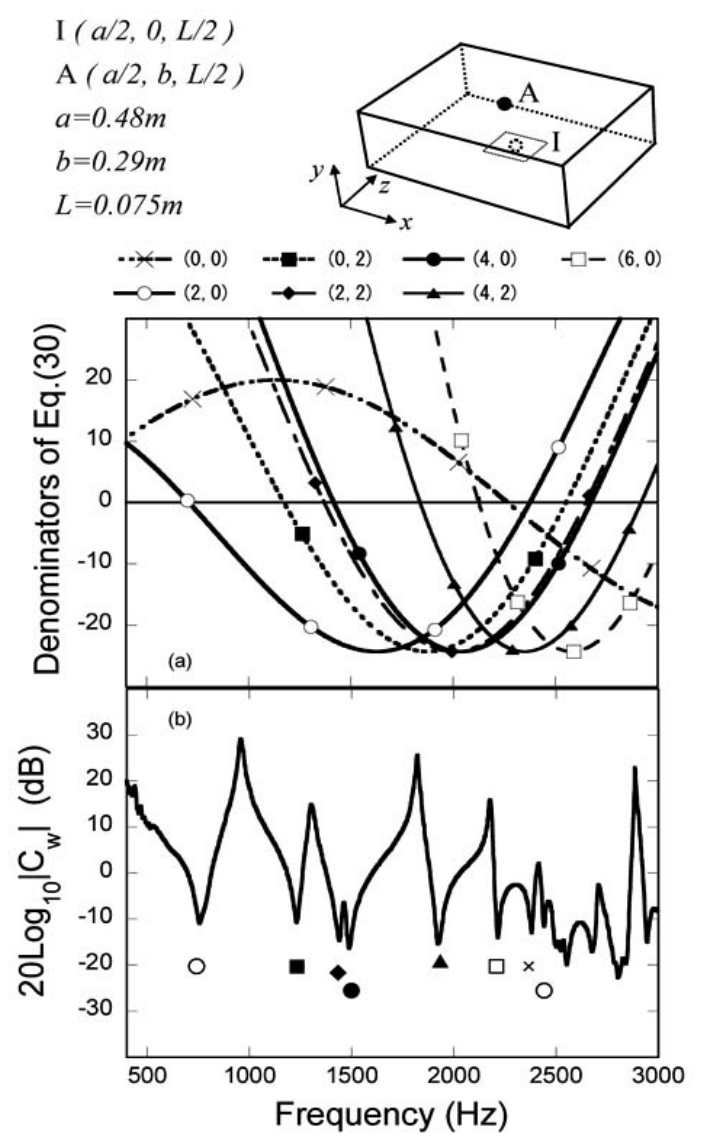

Fig. (11). Resonance frequencies appear in the cavity when the output is located at position A with changing input location (a) computed result of the denominator of Eq. (30). (b) measured result of $C_{w}$.

Table 2. The First Resonance Frequency of Some (m,n) Mode Occur in the Cavity when $a=0.48 \mathrm{~m}, b=0.29 \mathrm{~m}$ and $\mathbf{L}=\mathbf{0 . 0 7 5 m}$

\begin{tabular}{|c|c|c|}
\hline \multicolumn{2}{|c|}{ Mode } & $\mathbf{1}^{\text {st }}$ Resonance \\
\hline $\mathbf{m}$ & $\mathbf{n}$ & Frequency (Hz) \\
\hline \hline 1 & 0 & 369 \\
\hline 0 & 1 & 612 \\
\hline 1 & 1 & 715 \\
\hline 2 & 0 & 739 \\
\hline 2 & 1 & 960 \\
\hline 3 & 0 & 1109 \\
\hline 0 & 2 & 1224 \\
\hline 3 & 1 & 1267 \\
\hline 1 & 2 & 1278 \\
\hline 2 & 2 & 1430 \\
\hline 4 & 0 & 1479 \\
\hline 4 & 1 & 1600 \\
\hline 3 & 2 & 1652 \\
\hline
\end{tabular}




\section{CONCLUSIONS}

The characteristic of sound propagation in the rectangular ventilation having an input and output located at various positions, has been presented by solving the wave equation, considering the higher-order mode effects. Based on the obtained results, the cause and mechanism of resonance frequencies of parameter $\mathrm{C}$ are discussed in detail. To prove the theory, experiments with various positions were carried out and excellent agreement is obtained. The formulas derived from the present study enable the account of the insertion loss of the ventilation in practical applications. Also, it will be suitable for further study on the determination of optimized positions of input and output. This technology will be presented in an upcoming report.

\section{APPENDIX}

A block diagram of the whole experimental apparatus to measure the parameter $C_{w}$ is shown in Fig. (12) [7]. Two microphones were located at both sides of the ventilation unit to measure the sound pressures $P_{A}$ and $P_{B}$. Relationship between $P_{A}$ and $P_{B}$ is given by

$\left[\begin{array}{c}P_{A} \\ U_{A}\end{array}\right]=\left[\begin{array}{cc}\cos k l_{0} & j Z_{0} \sin k l_{0} \\ j \frac{1}{Z_{0}} \sin k l_{0} & \cos k l_{0}\end{array}\right]\left[\begin{array}{cc}A_{w} & B_{w} \\ C_{w} & D_{w}\end{array}\right]\left[\begin{array}{c}P_{B} \\ U_{B}\end{array}\right]$

where the first term and the second term represent the fourpole parameters of the input pipe which has length $l_{0}$ and the ventilation unit, respectively. Symbols $U_{A}, U_{B}$ and $Z_{0}$ represent velocity of the volume and acoustic impedance of the input pipe. By installing the microphone 2 on the output piston, $U_{B}$ will become zero, therefore Eq. (A-1) can be written as

$$
P_{A}=\left(A_{w} \cos k l_{0}+C_{w} Z_{0} \sin k l_{0}\right) P_{B}
$$

Furthermore, since the first term can be disregarded as compared to the second term, Eq. (A-2) can be written in terms of $\mathrm{dB}$ as follows

$$
20 \log _{10}\left|C_{w}\right|=20 \log _{10}\left|P_{A} / P_{B}\right|-20 \log _{10}\left|Z_{0} \sin k l_{0}\right|
$$

This means $C_{w}$ can be obtained by subtracting the acoustic characteristic of the input pipe $Z_{0} \sin k l_{0}$ from the measured sound pressures $P_{A}$ and $P_{B}$.

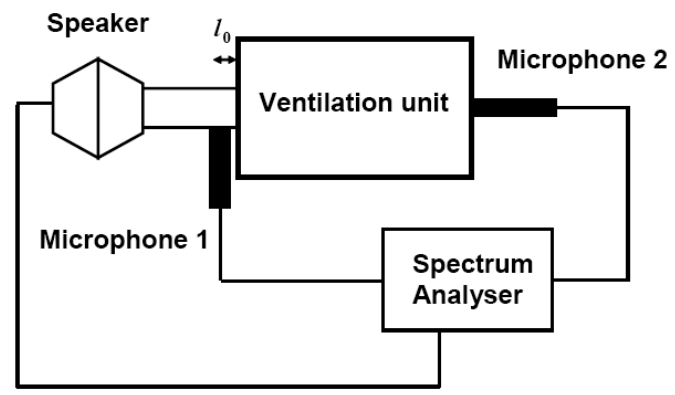

Fig. (12). Block diagram of the experimental apparatus.

\section{REFERENCES}

[1] Nishimura Y, Nishimura S, Nishimura T, Yano T. Sound propagation in soundproofing casement windows. J Appl Acoust 2009; 70(2009): 1160-7.

[2] Ih J. G. The reactive attenuation of rectangular plenum chambers. J Sound Vib 1992; 157(1): 93-122.

[3] Eriksson LJ. Higher order mode effects in circular ducts and expansion chambers. J Acoust Soc Am 1980; 68(2): 545-50.

[4] Hansen Z. Active control of higher order acoustic modes in ducts. J Acoust Soc Am 1992; 92(1): 244-57.

[5] Yin Y, Horoshenkov. The attenuation of the higher-order crosssection modes in a duct with a thin porous layer. J Acoust Soc Am 2005; 117(2): 528-35

[6] Munjal ML. Acoustics of Ducts and Mufflers. New York: Willey 1987.

[7] Nishimura S, Nishimura T, Yano T. Acoustic analysis of elliptical muffler chamber having a perforated pipe. J Sound Vib 2006; 297: 761-73. 